\title{
The dynamics of multiple pair-wise collisions in a chain for designing optimal shock amplifiers
}

\author{
Bryan Rodgers ${ }^{\mathrm{a}, *}$, Suresh Goyal ${ }^{\mathrm{b}}$, Gerard Kelly ${ }^{\mathrm{a}}$ and Michael Sheehy ${ }^{\mathrm{a}}$ \\ ${ }^{a}$ CTVR, Stokes Institute, University of Limerick, Limerick, Ireland \\ ${ }^{\mathrm{b}}$ Bell Labs Ireland, Alcatel-Lucent, Blanchardstown Industrial Park, Dublin 15, Ireland
}

Received 21 February 2008

Revised 14 May 2008

\begin{abstract}
The major focus of this work is to examine the dynamics of velocity amplification through pair-wise collisions between multiple masses in a chain, in order to develop useful machines. For instance low-cost machines based on this principle could be used for detailed, very-high acceleration shock-testing of MEMS devices. A theoretical basis for determining the number and mass of intermediate stages in such a velocity amplifier, based on simple rigid body mechanics, is proposed. The influence of mass ratios and the coefficient of restitution on the optimisation of the system is identified and investigated. In particular, two cases are examined: in the first, the velocity of the final mass in the chain (that would have the object under test mounted on it) is maximised by defining the ratio of adjacent masses according to a power law relationship; in the second, the energy transfer efficiency of the system is maximised by choosing the mass ratios such that all masses except the final mass come to rest following impact. Comparisons are drawn between both cases and the results are used in proposing design guidelines for optimal shock amplifiers. It is shown that for most practical systems, a shock amplifier with mass ratios based on a power law relationship is optimal and can easily yield velocity amplifications of a factor 5-8 times. A prototype shock testing machine that was made using above principles is briefly introduced.
\end{abstract}

\section{Nomenclature}

$\begin{array}{lll}\text { Symbol } & \text { Description } & \text { Units } \\ A_{i} & \text { Velocity amplification for mass } i & - \\ e_{j, i} & \text { Coefficient of restitution between masses } j \text { and } i & - \\ g_{j, i} & \text { Initial velocity ratio of masses } j \text { and } i & - \\ G_{n} & \text { Velocity amplification for system } & - \\ K_{n} & \text { Kinetic energy transfer efficiency for system } & - \\ m_{i} & \text { Mass of mass } i & \mathrm{~kg} \\ M & \text { Total system mass } & \mathrm{kg} \\ r_{j, i} & \text { Mass ratio of masses } j \text { and } i & - \\ V_{i} & \text { Velocity of mass } i \text { (after impact) } & \mathrm{m} / \mathrm{s} \\ v_{i} & \text { Velocity of mass } i \text { (before impact) } & \mathrm{m} / \mathrm{s}\end{array}$

\footnotetext{
*Corresponding author. Tel.: +35318082471; E-mail: bryan.rodgers@enterprise-ireland.com.
} 


$\begin{array}{ll}\text { Subscripts } & \\ f f & \text { Free-fall } \\ 1,2, \ldots, i, \ldots, n & \text { Mass number } \\ M E T & \text { Maximum energy transfer } \\ M V G & \text { Maximum velocity gain } \\ \text { Superscripts } & \\ \max & \text { Optimum or maximum }\end{array}$

\section{Introduction}

This paper examines the dynamics of velocity amplification through collisions between multiple masses with a view to developing useful machines. There are two kinds of applications of impacts between multiple objects: (1) Building machines that amplify shock or velocity like shock testers, ballistic launchers etc., and (2) Developing mechanisms and materials for absorbing shock or energy like protective armour, and shock protection of portable objects [4]. The dynamics principles underlying both of the above are the same. The current work will focus more on velocity amplification and develop a set of rules and guidelines for developing versatile and low-cost machines for very-high shock testing of emerging electronic components like MEMS, nano-optics devices, and sensors. In most cases, however, the design rules could be equally applicable to developing shock absorbers.

Due to their small masses, packaged MEMS devices can be designed to be very rugged. They are commonly found in telecommunication equipment with very high reliability requirements; and in critical missile control systems, smart ammunition and in space rockets, where they must be capable of performing reliably under severe acceleration conditions, perhaps following a long period of storage. Current test methods used for testing shock-hardened MEMS devices include the Hopkinson bar [1], rail and air guns, very-high drop testing, pneumatic shock and centrifuging [11].

The shock testing machines being proposed in this paper, based on velocity/shock amplification through multiple impacts, offer several advantages over the above methods, including:

- They offer detailed and precise testing capabilities using a single machine that allows a vast range of shock pulses so that shock testing can be incorporated early in the design stage of the device.

- Such machines can be scaled with the size of the object being tested.

- They can be used in stand-alone mode or as attachments to existing shock testing machines, thereby vastly increasing their capability.

- They enable high-speed video recording of the device during test to be implemented relatively easily.

- They provide safer and much lower-cost shock testing capability.

In the late 1960s and early 1970s, a number of papers were published that analysed the dynamics of multiple impacts and associated velocity amplification [6-8]. In their paper on energy transfer in one-dimensional collisions of multiple objects, Hart and Hermann [6] showed that for maximum energy transfer between a moving mass and the final mass in a chain of several stationary masses, each intermediate mass should be a geometric mean of its adjacent masses. Kerwin [8] looked at a similar system and deduced the equations for velocity, momentum and kinetic energy transfer for cases where the coefficient of restitution is either one or zero. He also used a velocity transformation to apply the results to a system of successively smaller vertically-stacked masses dropped on the ground. Harter et al. [7] studied the behaviour of three successively smaller rubber balls stacked one above the other and dropped on a rigid surface. They analysed this system using elastic and inelastic independent-collision models, and quasi-linear and non-linear elastic continuous force models. They predicted the ratio between successive masses in the case of an elastic system (where the coefficient of restitution is unity) such that all masses, except the final one, come to rest after the collisions. They also showed that the velocity of the final mass in this case varies linearly with the number of masses in the system.

Recently other authors $[9,10]$ have analysed similar systems using a variety of more sophisticated approaches. Patrício [9] used Hertzian contact theory to develop equations of motion for two masses impacting a rigid plane and showed that significantly non-rigid impacts lead to more complicated interactions between the colliding masses, like 


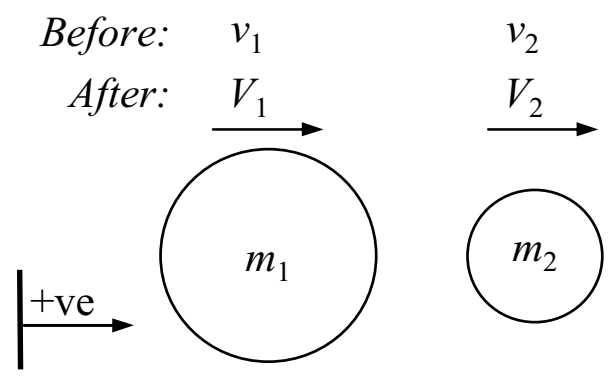

Fig. 1. System of two impacting masses.

all three bodies impacting at once, resulting in velocity amplifications that are much lower than those predicted by the independent-collisions model. Pöschel and Brilliantov [10], in perhaps the most thorough treatment of this topic using the independent collision approach, showed that for an elastic system similar to that described by Hart and Hermann [6], the kinetic energy of the first mass is transferred completely to the last mass if there are an infinite number of intermediate masses. They also proposed a method for calculating the optimum number of masses in a chain where each collision is dissipative and the coefficient of restitution is a constant less than one. Finally, they developed theories for the case of visco-elastic bodies where the coefficient of restitution is not constant, but a function of the impact velocity.

The manner in which the current work differs from previous work is primarily in its focus on developing guidelines for building useful machines. Besides gathering all the relevant analytical results from previous work into one place, the novelty centres around the deeper analysis of the various configurations of shock amplifiers mentioned in the paper, comparisons of their relative merits, and the development of design guidelines.

All the analyses presented in the paper are based on simple rigid body models - primarily those governing the momentum transfer and energy dissipation during impacts between two point masses or rigid spheres. These are sufficient because, a) the shock machines being discussed in this paper are designed to ensure independent pair-wise collisions, b) the guidelines being developed are based on velocity amplifications and not acceleration levels so the details of every impact are not important, only the velocities of the masses before and after impact, and c) the general results are valid despite deviations from rigid-body models.

The layout of the rest of the paper is as follows: the dynamics of impact between two masses is examined in detail followed by the dynamics of impacts between a chain of masses configured and stacked (both horizontally and vertically) for velocity amplification. Optimal design principles for vertically-stacked shock amplifiers are then determined followed by discussion and conclusions.

\section{Dynamics of impact between two masses}

Lots of useful machines can be conceptualized and designed by extrapolating the results of an impact between two masses, hence a thorough understanding of their dynamics is crucial. This section summarizes the dynamics of impact between masses $m_{1}$ and $m_{2}\left(m_{1}>m_{2}\right)$, illustrated in Fig. 1, using rigid body mechanics. Energy loss is modelled through the coefficient of restitution [2,3], and the influence of external forces during impact, including gravity, is neglected [5].

Conservation of momentum for the system implies that:

$$
m_{1} V_{1}+m_{2} V_{2}=m_{1} v_{1}+m_{2} v_{2}
$$

The coefficient of restitution $e_{2,1}$ for impact between $m_{2}$ and $m_{1}$ is defined as:

$$
e_{2,1}=-\frac{V_{2}-V_{1}}{v_{2}-v_{1}}
$$

Combining Eqs (1) and (2) leads to the following equations for the velocity gains of the masses after impact: 


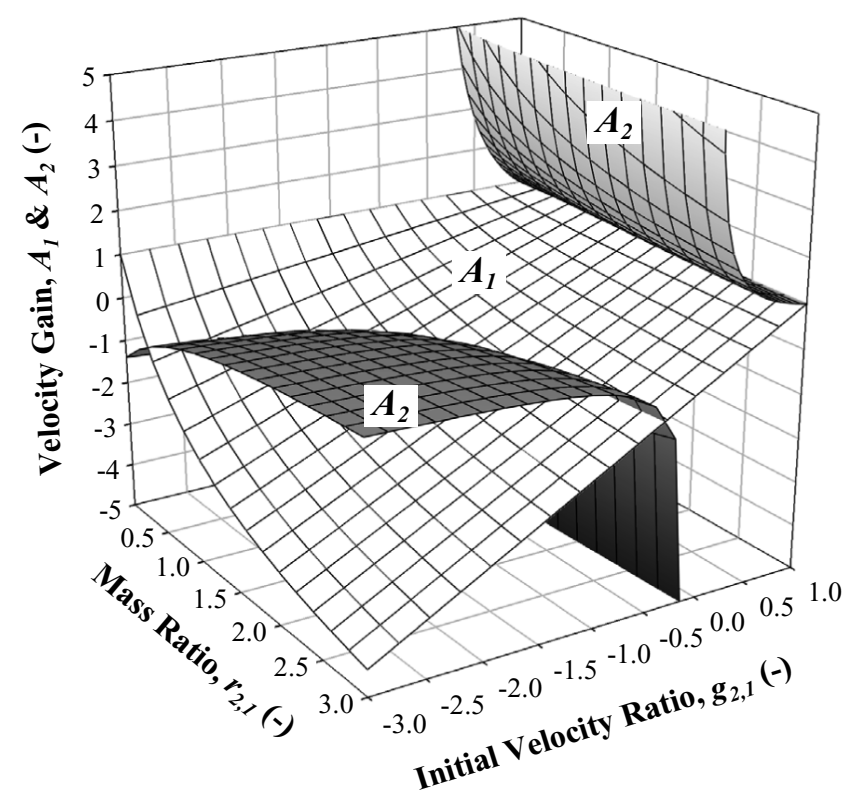

Fig. 2. 3D plot of velocity gain of masses $m_{1}$ and $m_{2}$ versus mass ratio and initial velocity ratio, for $r_{2,1}=0.8$. (-) means non-dimensional.

$$
\begin{aligned}
& A_{1}=\frac{V_{1}}{v_{1}}=1+\frac{r_{2,1}}{1+r_{2,1}}\left(1+e_{2,1}\right)\left(g_{2,1}-1\right) \\
& A_{2}=\frac{V_{2}}{v_{2}}=1-\frac{1}{1+r_{2,1}}\left(1+e_{2,1}\right)\left(1-\frac{1}{g_{2,1}}\right)
\end{aligned}
$$

where mass ratio $r_{2,1}$ and initial velocity ratio $g_{2,1}$ are defined as:

$$
\begin{aligned}
& r_{2,1}=\frac{m_{2}}{m_{1}} \\
& g_{2,1}=\frac{v_{2}}{v_{1}}
\end{aligned}
$$

If the velocity gains of both masses are plotted from Eqs (3) and (4), the resulting surfaces are as shown in Fig. 2 (for a representative high coefficient of restitution for real materials, $e_{2,1}=0.8$ ). Note that the range of initial velocity ratio is limited such that an impact will always occur (i.e., $-\infty<g_{2,1}<1$ ).

Figure 2 may be used to determine what happens to $m_{1}$ or $m_{2}$ following impact, both qualitatively and quantitatively, i.e. whether their velocity increases or decreases and the magnitude of the velocity change. (Note that given its definition, the velocity gain of the second mass tends to infinity for cases where $m_{2}$ starts out at rest.) For instance, regions of design space where $A_{2}>1$ represent conditions that lead to velocity amplification of $m_{2}$. One can use Fig. 2 further to reason about pair-wise impacts involving multiple masses. For instance, in a system of three masses with defined initial velocities and masses, the final velocity $V_{2}$ of $m_{2}$ after its impact with $m_{1}$ becomes its initial velocity $v_{2}$ in the subsequent collision with $m_{3}$.

Impacts involve momentum and energy transfer between the colliding objects. One special case of a two-mass impact occurs when one of the masses comes to rest after impact, thereby transferring all its momentum to the other mass. In other words, given the coefficient of restitution, such impacts maximise the transfer of kinetic energy from one mass to the other. Machines exploiting momentum-transferring impacts are germane to the current study, and the dynamics of these impacts is now examined further.

For $m_{1}$ to come to rest after impact $\left(V_{1}=0\right)$, it may be shown from Eq. (3) that the initial velocity ratio must be related to the coefficient of restitution and the mass ratio as follows: 


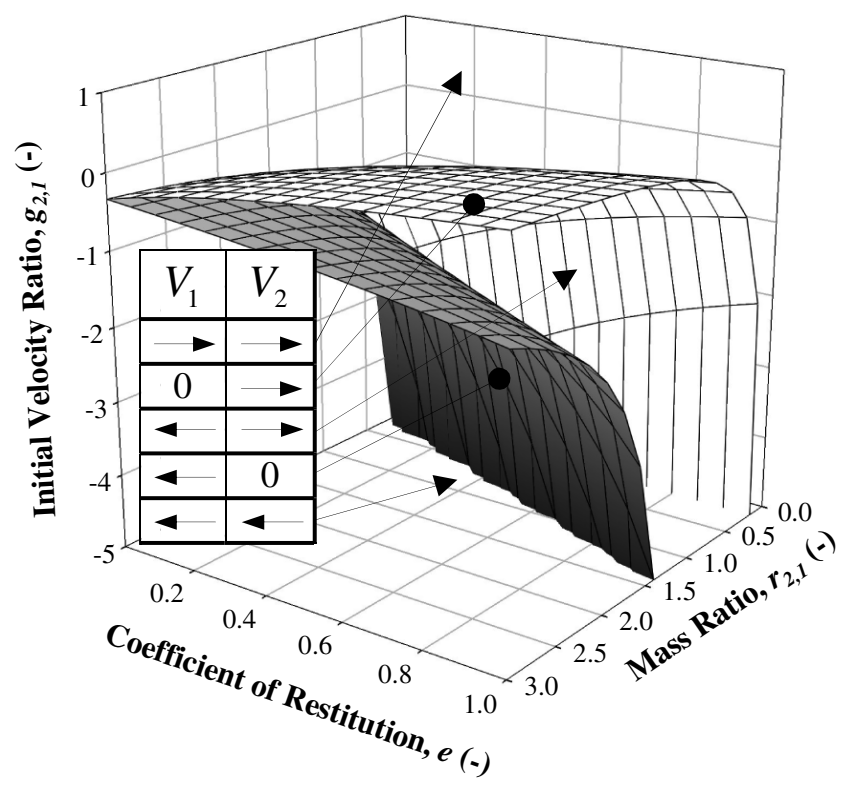

Fig. 3. 3D plot of initial velocity ratio, $g_{2,1}$ versus coefficient of restitution, $e_{2,1}$ and mass ratio, $r_{2,1}$ for the case where $V_{1}=0$ for the upper surface and $V_{2}=0$ for the lower surface. The table arrows indicate the direction of motion after impact. (-) means non-dimensional.

$$
g_{2,1}=\frac{e_{2,1}-1 / r_{2,1}}{1+e_{2,1}}
$$

Similarly, if $m_{2}$ comes to rest after impact ( $V_{2}=0$ ), it can be shown from Eq. (4) that:

$$
g_{2,1}=\frac{1+e_{2,1}}{e_{2,1}-r_{2,3}}
$$

Figure 3 plots Eqs (7) and (8), and it is useful in determining the direction of either of the two masses after impact. For all points on the surfaces in Fig. 3, at least one of the masses is at rest after impact. For any point below the lower surface in Fig. 3, both masses move in the negative direction after impact. At all points that lie between the surfaces, the masses move in opposite directions after collision. Finally, for any point that lies above the plotted surfaces, each mass moves in the positive direction after impact. The upper surface and the region above it is the design space most suitable for velocity or shock amplifiers.

A specialized case of maximal energy transference involves an impact between a moving object and a stationary object. Setting $V_{1}=v_{2}=0$ in Eqs (1) and (2), it may be shown that the velocity gain of the system (here defined as $G_{2}$ ) is given by:

$$
G_{2}=\frac{V_{2}}{v_{1}}=\frac{1}{r_{2,1}}=e_{2,1}
$$

Since the velocity gain of the system is equal to $e_{2,1}$ (which has an upper limit of one for a perfectly elastic collision), this arrangement is therefore not useful in amplifying velocity. However, it could be effective as a velocity attenuator or energy absorber for low coefficients of restitution.

\subsection{Kinetic energy transfer efficiency}

To formalize the investigation of the efficiency of kinetic energy transfer through collisions in a system of masses, a new parameter $K$ is introduced that is defined as the ratio of kinetic energy of a mass after collision to that of the kinetic energy of the total system before impact. Therefore, for mass one:

$$
K_{1}=\frac{\frac{1}{2} m_{1} V_{1}^{2}}{\frac{1}{2} m_{1} v_{1}^{2}+\frac{1}{2} m_{2} v_{2}^{2}}=\frac{\left(1+\frac{r_{2,1}}{1+r_{2,1}}\left(1+e_{2,1}\right)\left(g_{2,1}-1\right)\right)^{2}}{1+r_{2,1} g_{2,1}^{2}}
$$




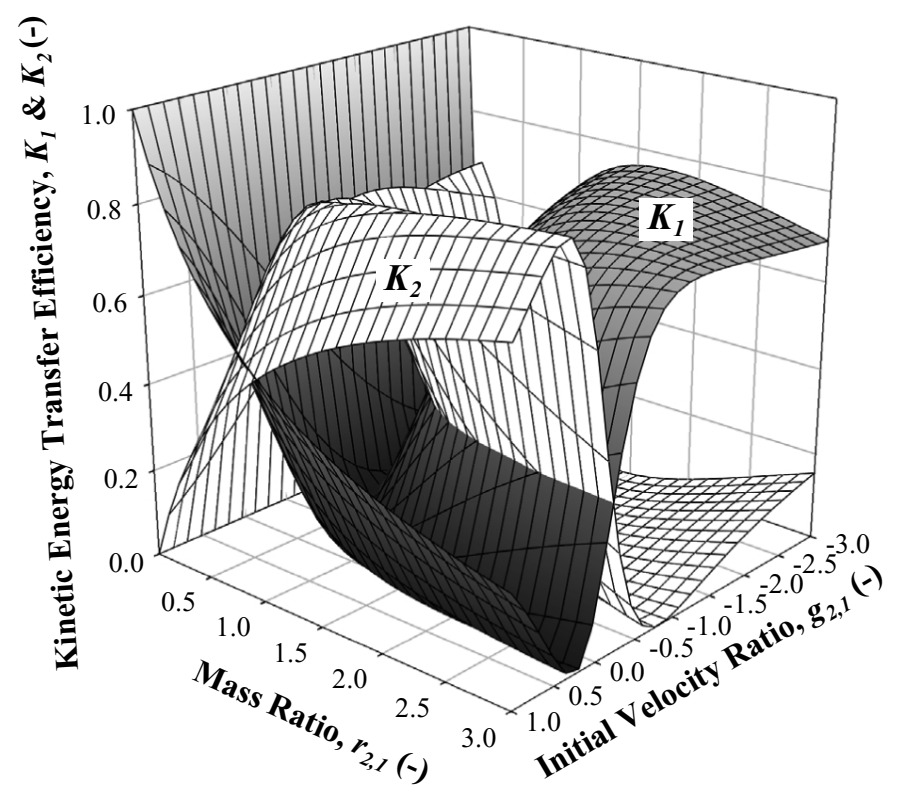

Fig. 4. 3D Plot of kinetic energy transfer efficiency of both masses after impact over initial system energy versus initial velocity ratio and mass ratio for $e_{2,1}<0.8$. (-) means non-dimensional.

Similarly, for the second mass:

$$
K_{2}=\frac{r_{2,1}\left(g_{2,1}-\frac{1}{1+r_{2,1}}\left(1+e_{2,1}\right)\left(g_{2,1}-1\right)\right)^{2}}{1+r_{2,1} g_{2,1}^{2}}
$$

The sum of these kinetic energy transfer efficiencies will be one when the coefficient of restitution is one (i.e. when there is no energy lost in the collision). For the special case where the second mass is initially at rest (i.e. $g_{2,1}=0$ ), the energy efficiency reduces to:

$$
K_{2}=\frac{r_{2,1}\left(1+e_{2,1}\right)^{2}}{\left(1+r_{2,1}\right)^{2}}
$$

a result also found by Hart and Herrmann [6].

Figure 4 displays the equations for kinetic energy transfer efficiency ratio as a function of initial velocity ratio and mass ratio (again for the high coefficient of restitution of 0.8). Note that since $e_{2,1}<1, K_{1}+K_{2}<1$.

\section{Shock amplification through a series of horizontal masses}

One of the main inferences from Section 2 is that a heavy mass impacting a reasonably light mass, with a sufficiently high coefficient of restitution, can cause a velocity amplification of the lighter mass. For instance in a perfectly elastic collision between two masses that approach each other with equal and opposite velocities, with one of the masses being at least three times as heavy as the other one, the lighter mass rebounds with a velocity that is more than twice its velocity before impact.

Therefore a natural configuration to consider for a velocity amplifier or launcher is that of a series of progressively and appropriately smaller masses in the horizontal plane as shown in Fig. 5. All the masses are initially at rest except for the largest mass $m_{1}$, which impacts the second mass $m_{2}$, which impacts $m_{3}$ and so on until the last mass $m_{n}$ is impacted. Depending on the mass ratios between the successive masses of the chain in Fig. 5, and the coefficient of restitution, mass $m_{n}$ could be launched with significantly higher velocity than that of $m_{1}$.

It can be shown that the general equation for the velocity gain $G_{n}$ of the final mass after impact is: 


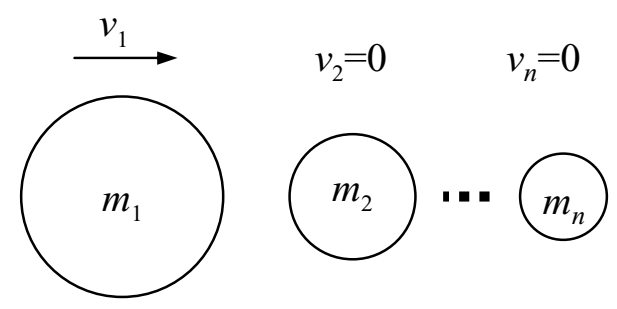

Fig. 5. System of sequentially smaller, stationary masses impacted by a moving mass.

$$
G_{n}=\prod_{k=2}^{n}\left(\frac{1+e_{k, k-1}}{1+r_{k, k-1}}\right)
$$

This result is similar to that found by Kerwin [8].

\subsection{Maximum velocity gain system}

For a fixed kinetic energy input into the system in Fig. 5 (i.e., given mass $m_{1}$ and velocity $v_{1}$ ) and a given final mass $m_{n}$, the question arises if there is a configuration of the system that maximizes the velocity $V_{n}$ of $m_{n}$.

Given $m_{1}$ and $m_{n}$ implies that the product of the pair-wise mass ratios of the masses in Fig. 5 is fixed, no matter how many masses there are in the chain, because:

$$
R=\frac{m_{n}}{m_{1}}=r_{2,1} \cdot r_{3,2} \cdot r_{4,3} \ldots r_{n, n-1}
$$

Hart and Hermann [6], Kerwin [8], Pöschel and Brilliantov [10], and others showed that for a constant coefficient of restitution $e$ for all the impacts and a given $n$, the velocity gain of $m_{n}$ is maximised by choosing the following power law relationship to define the pair-wise mass ratios:

$$
r_{i, i-1}=R^{\frac{1}{n-1}} \quad(i=2,3, \ldots, n)
$$

And the corresponding maximum velocity gain of $m_{n}$ is given by:

$$
G_{n}=\frac{V_{n}}{v_{1}}=\left[\frac{1+e}{1+R^{\frac{1}{n-1}}}\right]^{n-1}
$$

In addition, the kinetic energy transfer efficiency for the last mass is given as [6],

$$
K_{n}=\frac{R(1+e)^{2(n-1)}}{\left(1+R^{\frac{1}{n-1}}\right)^{2(n-1)}}
$$

A chain of $n$ masses with mass ratios specified by Eq. (15) is referred to as the Maximum Velocity Gain (MVG) system in the rest of this paper. It is clear from Eqs (15) and (16) that the mass distribution of an MVG system is independent of the coefficient of restitution but its velocity gain is not. It can be shown from equation (16) that for perfectly elastic collisions (i.e., $e=1$ ) the velocity gain continues to increase with increasing number of masses in the chain, approaching an asymptotic limit for $n \rightarrow \infty$, and defined as:

$$
\underset{n \rightarrow \infty}{G_{n}}=\frac{1}{\sqrt{R}}
$$

Equation (18) implies that with an infinite chain of elastic collisions, all the energy of the first mass is transferred to the last mass.

For inelastic collisions (i.e., $e<1$ ), however, there is a trade-off between the velocity gain and the energy loss by an additional impact. Therefore the velocity gain does not increase monotonically with $n$, but rather it has a maximum value $G_{n}^{\text {max }}$ for a given $e$. In addition, as supported by intuition, $G_{n}^{\max }$ decreases with decreasing $e$. Pöschel and Brilliantov [10] proposed a numerical solution to Eq. (16) for determining $G_{n}^{\max }$ and the corresponding $n^{\max }$ for a given $e$, although it is possible to get $G_{n}^{\max }$ and $n^{\max }$ more directly by simply tabulating or plotting Eq. (16). 


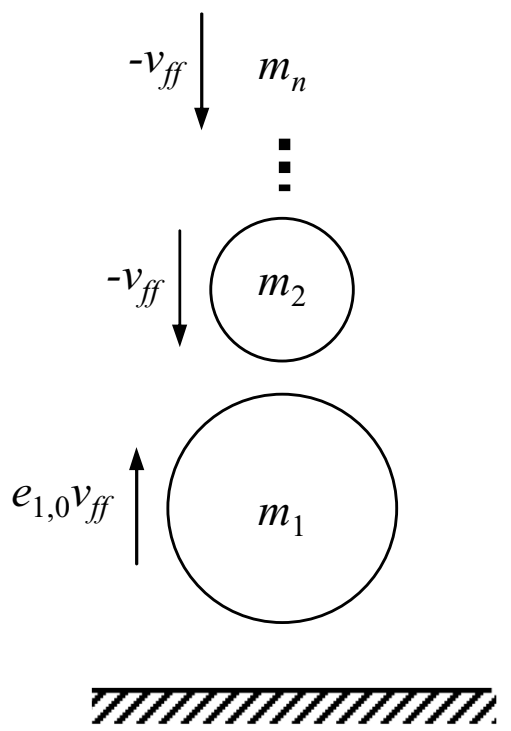

Fig. 6. Model of velocity amplifier prior to second impact.

\section{Shock amplification through a series of vertically-stacked masses}

In general, shock-testing machines (or drop-testing machines) tend to be vertical in order to use gravity to create impact velocity. Similarly, gravity can be used to advantage in designing a shock amplification system composed of a series of vertically-stacked masses, separated by an arbitrarily small distance from each other, as shown in Fig. 6. Like with the horizontal configuration discussed in the previous section, the heaviest mass is at the bottom of the chain with progressively smaller masses above it, so that $m_{1}>m_{2}>\ldots>m_{n}$. Assume that the entire chain is dropped from some height towards the ground such that initially all the masses are approaching the ground with the same free-fall velocity, $v_{f f}$. The sequence of events that occurs at impact, in terms of rigid-body mechanics, is as follows.

Mass $m_{1}$ impacts the ground first, instantaneously reversing its velocity to $e_{1,0} v_{f f}$ and then impacts the approaching mass $m_{2}$. With an appropriate $e_{2,1}$ and mass ratio $r_{2,1}$ as found from the equations in Section 2, $m_{2}$ will reverse its velocity with a magnitude significantly higher than its approach velocity of $v_{f f}$. This phenomenon of velocity amplification through pair-wise impacts continues sequentially as $m_{2}$ impacts $m_{3}$, which then impacts $m_{4}$, and so on until mass $m_{n-1}$ impacts the final mass in the chain $m_{n}$.

Note that with a change in reference frame, the system described above is the same as the horizontal system described in Section 3, with the heaviest mass approaching the rest of the stationary masses (in the new reference frame) with velocity $\left(1+e_{1,0}\right) v_{f f}$. Equation (19) then implies that for the vertically-stacked series of masses of Fig. 6, the general expression for the velocity gain of $m_{n}$, following the sequence of impacts described above, is:

$$
G_{n}=\left(1+e_{1,0}\right) \prod_{k=2}^{n}\left(\frac{1+e_{k, k-1}}{1+r_{k, k-1}}\right)-1
$$

Equation (19) describes the velocity gain for systems somewhat more general than that explained in Fig. 6, because by assuming values of $e_{1,0}>1$ we have systems where the first mass could hit an 'active ground' with the potential for adding energy to $m_{1}$.

\subsection{Maximum velocity gain system}

All the assumptions and computations leading to the horizontal configuration maximum velocity gain system, with mass ratios given by Eq. (15), are equally valid for the vertically-stacked system of masses. In other words, for a vertically-stacked chain of $n$ masses with constant coefficient of restitution $e$, the velocity gain of the topmost 


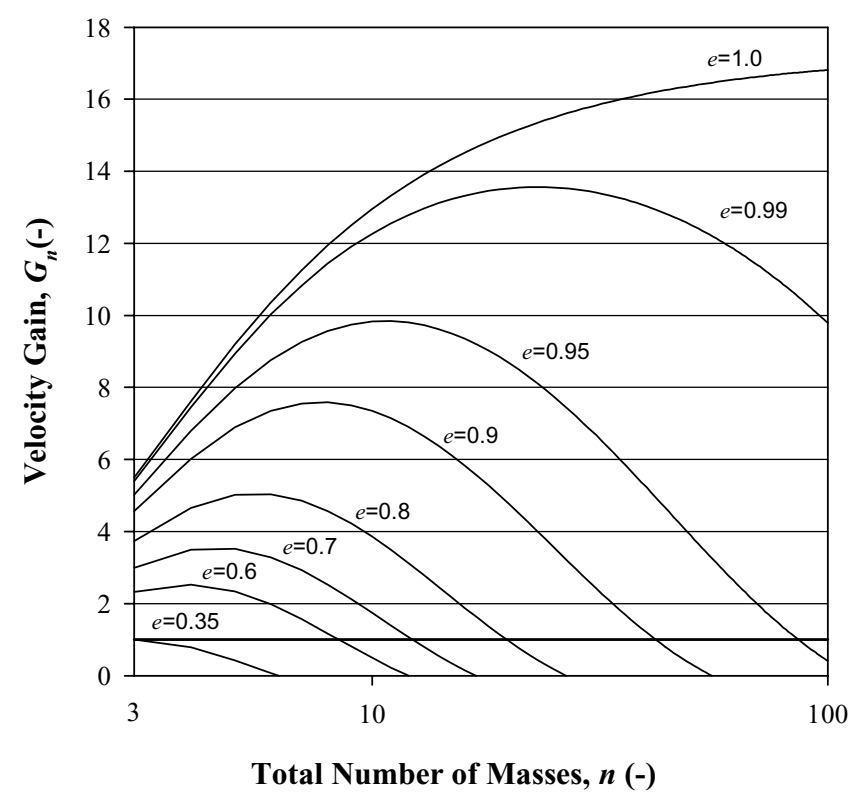

Fig. 7. Plot illustrating velocity gain as a function of the number of masses and the coefficient of restitution, for a vertically-stacked MVG chain with $R=0.012$. (-) means non-dimensional.

mass can be maximized if the mass ratios obey the power law of Eq. (15), and it is given as:

$$
G_{n}=\frac{(1+e)^{n}}{\left(1+R^{\frac{1}{n-1}}\right)^{n-1}}-1
$$

Further, for perfectly elastic collisions ( $e=1$ ), the asymptotic limit for velocity gain when $n \rightarrow \infty$, is given by:

$$
\underset{n \rightarrow \infty}{G_{n}}=\frac{2-\sqrt{R}}{\sqrt{R}}
$$

For inelastic collisions, for every given $e$, an $n^{\max }$ that leads to a $G_{n}^{\max }$ can be found directly by tabulating or plotting Eq. (20). $G_{n}^{\max }$ decreases with decreasing $e$. Figure 7 illustrates how the velocity gain changes with the number of masses in the chain, the presence of an $n^{\max }$ and a corresponding $G_{n}^{\max }$ and their dependence on $e$. Note that the maximum velocity amplification achievable through this scheme of multiple impacts decreases rapidly with the increase of energy dissipation associated with every impact, such that for values of $e<0.35$ there is no velocity amplification at all. Hence for purposes of building a shock-amplifier, it is imperative that the impacts be mediated through materials/methods that have very high restitution.

It may also be shown that $n^{\max }$ can be found by using similar equations proposed by Pöschel and Brilliantov [10] for the horizontal MVG case, using the following method. First determine

$$
n^{*}=\frac{\ln (R)}{\ln \left(x_{0}\right)}+1
$$

where $x_{0}$ is the solution to the equation:

$$
\left(1+x_{0}\right)=(1+e) x_{0}^{x_{0} / 1+x_{0}}
$$

and then calculate:

$$
G^{*}=\frac{(1+e)^{n^{*}}}{(1+R)^{n^{*}-1}}-1
$$




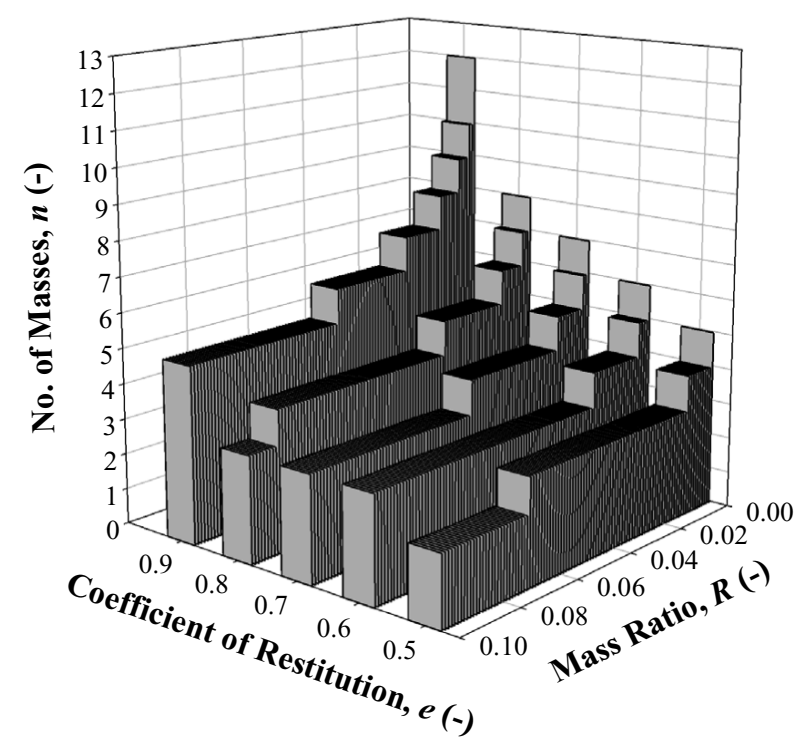

Fig. 8. Plot of optimum number of masses to maximise velocity of the uppermost mass versus mass ratio and coefficient of restitution, for a vertically-stacked MVG system. (-) means non-dimensional.

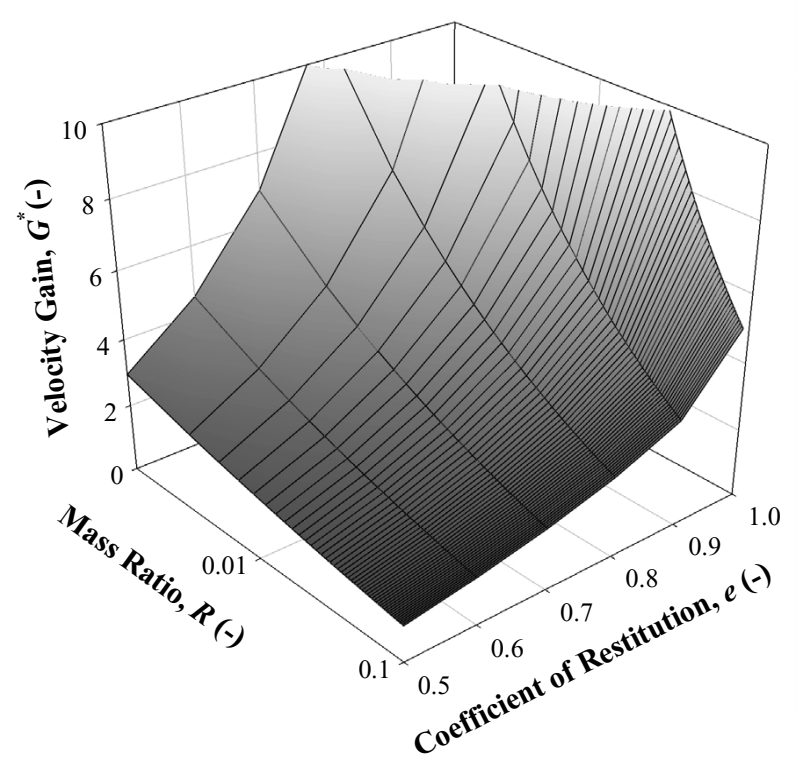

Fig. 9. Plot of $G^{*}$ versus $R$ and $e$, for the MVG system of vertically-stacked masses. (-) means non-dimensional.

The nearest rounded integer (both floor and ceiling) to $n^{*}$ that yields a higher value of $G_{n}$ through Eq. (24) is then $n^{\max }$ and the corresponding $G_{n}$ is $G_{n}^{\max }$. Figure 8 shows the value for $n^{\text {max }}$ computed for the range of $e=[0,1]$ and for a large range of $R$.

While designing a shock amplification machine for testing purposes, design constraints arise from the size of the machine, the size and shape of the objects to be tested, the materials that are available, the required shock levels and their durations, etc. The above, in turn, impose constraints $R$ on $e$. While Fig. 7 gives a sense of the velocity amplifications that are possible for $R=0.012$, Fig. 9 gives a broader picture of the dependence of $G^{*}$ (which is very close to $G_{n}^{\max }$ ) on $R$ and $e$. 
The plot indicates that $G^{*}$ increases monotonically with increasing $e$ and decreasing $R$. For high coefficient of restitution, the rapid increase in $G^{*}$ with decreasing mass ratio illustrates how effectively the velocity amplification principle can be utilized for building powerful velocity amplifiers.

\subsection{Maximum energy transfer system}

Since transferring energy from a heavier mass to a lighter mass through impact is the primary basis for the shock amplification being explored here, intuition suggests that a system of masses where all the energy is transferred to the lightest mass would be the most efficient one as a shock amplifier. Such a system composed of a chain of masses where all of them, except the final mass $m_{n}$, come to rest after impact is henceforth called as the Maximum Energy Transfer (MET) system. Extrapolating Eq. (9) shows that in the horizontal configuration, the MET system does not lead to velocity amplification. In the vertically-stacked configuration, however, the fact that all the masses start in a state of motion and pair-wise impacts occur between masses travelling in opposite directions leads to significant velocity amplification in the MET system.

Assuming a constant coefficient of restitution $e$ for all impacts, it can be shown that the mass ratios required for the vertically-stacked MET system have to satisfy the condition:

$$
r_{i+1, i}=\frac{\sum_{k=1}^{i} e^{k}}{1+\sum_{k=1}^{i+1} e^{k}}(i=1,2, \ldots, n-1)
$$

For elastic collisions, Eq. (25) reduces to [7]:

$$
r_{i+1, i}=\frac{i}{i+2} \quad(i=1,2, \ldots, n-1)
$$

For inelastic collisions $(e<1)$, it can be shown by induction that Eq. (25) reduces to:

$$
r_{i+1, i}=\frac{e^{i+1}-e}{e^{i+2}-1} \quad(e<1 ; i=1,2, \ldots, n-1)
$$

Further manipulation of Eq. (27) reveals that for larger values of $i$, it quickly reaches a limit given by:

$$
\lim _{i \rightarrow \infty} r_{i+1, i}=e
$$

Unlike with the power law in the MVG configuration, the mass ratios in the MET configuration are not the same along the chain and do not depend on $R$, i.e. the ratio of the first and the final mass. Instead, as shown by Eqs (25), (27) and (28), the mass ratios depend on $e$. Figure 10 plots the mass ratios to illustrate their dependence on $e$.

The velocity gain for the uppermost mass of the vertically-stacked MET can be derived to be:

$$
G_{n}=\frac{V_{n}}{v_{f f}}=\sum_{k=1}^{n} e^{k}
$$

It is clear from Eq. (29) that unlike the MVG system, for the MET system the velocity gain of the uppermost mass increases monotonically with $n$ and $e$. For elastic collisions, Eq. (29) reduces to $G_{n}=n$. Although this implies that velocity gain increases without limit for elastic collisions, it is also clear from Eq. (29) that with inelastic collisions the achievable velocity gain from a chain of vertically-stacked masses is limited, with $G_{n}$ quickly approaching a limit given by:

$$
\lim _{n \rightarrow \infty} G_{n}=G_{n}^{\max }=\frac{e}{1-e}
$$

Equation (30) implies that in a vertically-stacked MET system, velocity gain (i.e., $G_{n}>1$ ) is only achievable if $e>0.5$. (Note that this is higher than the requirement for an MVG system where velocity gain can be attained as long as $e>0.35$ ). Equation (29) is plotted in Fig. 11, for masses ranging from two to ten in the chain, to further 


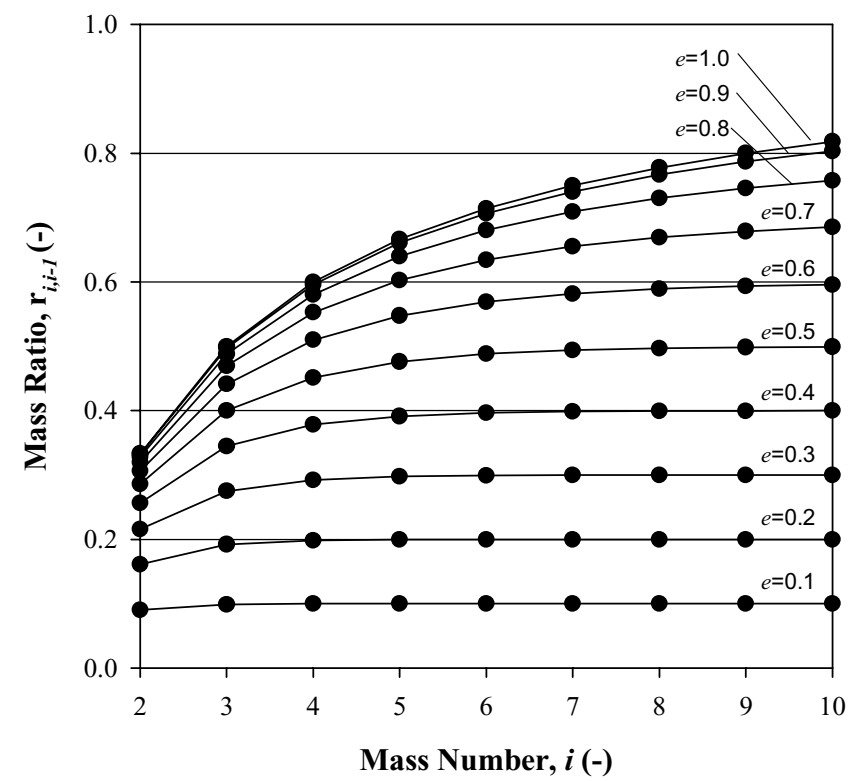

Fig. 10. Pair-wise mass ratios $r_{i, i-1}$ versus mass number for varying coefficient of restitution in a vertically-stacked MET configuration. (-) means non-dimensional.

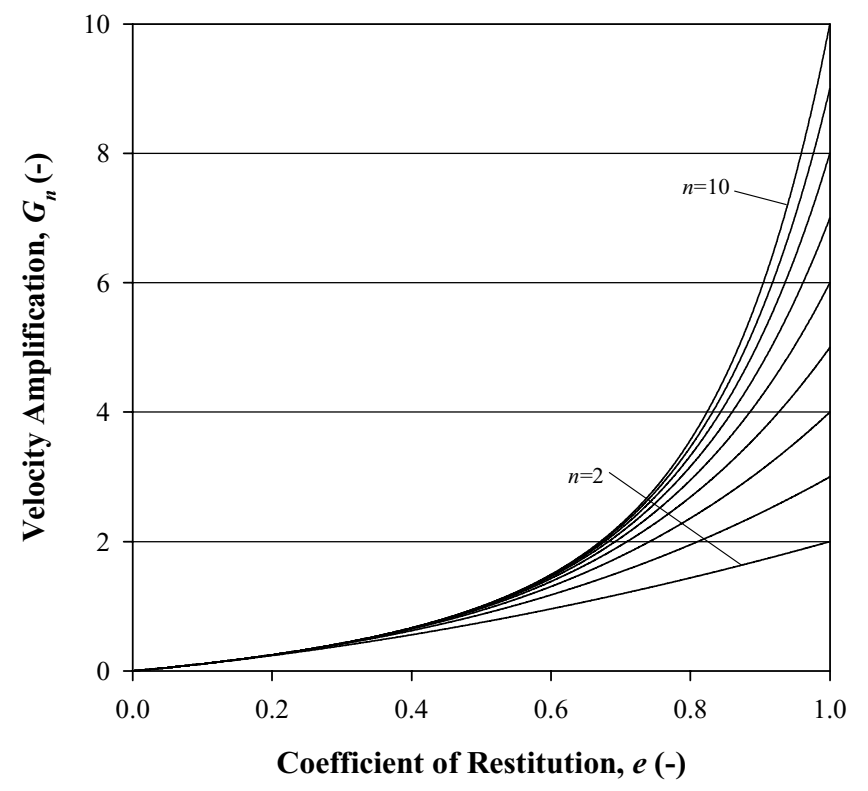

Fig. 11. Velocity amplification of the uppermost mass in vertically-stacked MET chains, consisting of two to ten masses, as a function of coefficient of restitution. (-) means non-dimensional.

illustrate that for values of $e$ significantly less than $1, G_{n}^{\max }$ is modest and achieved with fairly short chains. For more dramatic velocity gains that realize the benefit of somewhat longer chains, it is necessary to have high values of $e$.

Figure 12 plots the mass ratios $m_{i} / m_{1}$ for every mass in a MET chain, and also illustrates how the plots of Fig. 12 can be used to facilitate the design of a vertically-stacked MET shock amplifier. For instance if $m_{n}, m_{1}$ and $e$ are known, the mass ratio $m_{n} / m_{1}$ can be calculated, and identified as a point on the appropriate $e$ curve in Fig. 12. All 


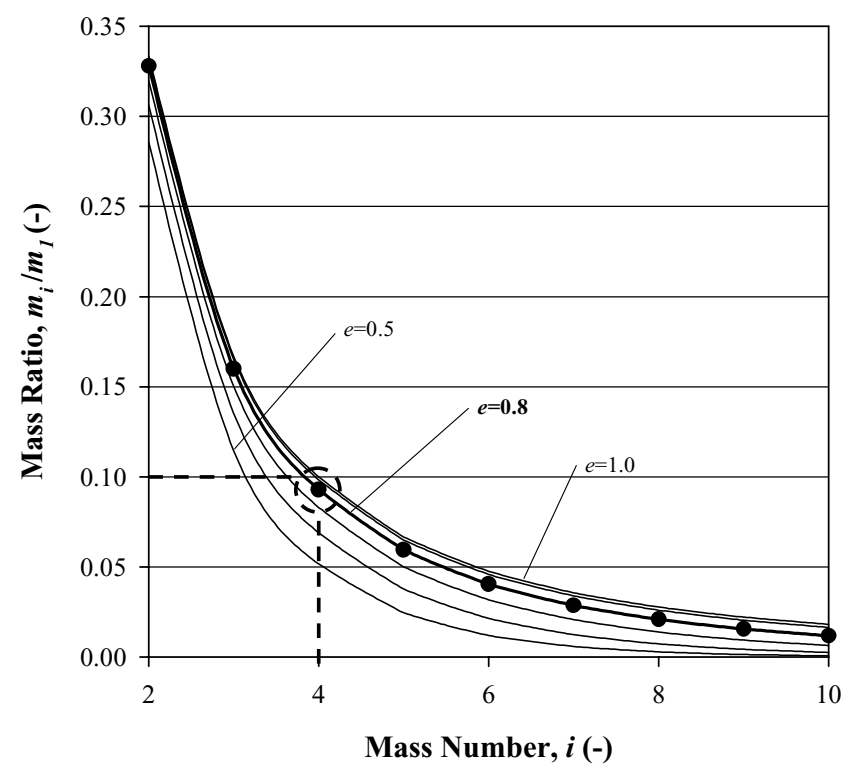

Fig. 12. Plot of mass ratio $m_{i} / m_{1}$ versus mass number for varying coefficient of restitution in a vertically-stacked MET configuration. (-) means non-dimensional.

the mass ratios higher than $m_{n} / m_{1}$, and corresponding to integer points for mass number, are the mass ratios to be used for intervening masses in the vertically-stacked MET chain. If only $e$ and $m_{1}$ are known, but a certain $G_{n}$ is desired, the number of masses needed in the chain can be figured out from the appropriate curve in Fig. 11 and their mass ratios from Fig. 12. For instance in the example illustrated in Fig. 12 - determining the number of masses for a machine with $R=0.1$ - the nearest whole number is 4 .

\section{The optimal drop testing configuration}

Given that both MET and MVG systems are capable of providing very significant velocity amplification in a vertically-stacked configuration, the next issue is whether there are regions in 'design space' where one of them is more optimal over the other for a practical shock amplifier. Generally the design goals for a vertically-stacked shock amplifier are to maximize the velocity gain and the kinetic energy transfer efficiency for the top-most mass while minimizing the total mass in the system for a given $R$ and $e$. The proposed amplifier consists of a series of successively smaller masses stacked vertically and dropped on a rigid surface such that a sequence of impacts occurs between the masses and the uppermost mass achieves a large gain in velocity. In practice the masses will be guided using linear bearings running on rods so that motion is confined to the vertical axis.

Accordingly Figs 13, 14 and 15 compare the velocity gain, kinetic energy transfer efficiency and the total system mass respectively for a very large range of $R$ and $e$ (i.e., a large part of the practically encountered design space) for the MVG and the MET configurations. The comparisons are done by normalizing the values of the above three metrics for the MVG system by the corresponding values for the MET system. Note that for any given $R$ and $e$ in these figures, the values of $G_{n}, K_{n}$ and $M$ for the MVG system are those of an optimal configuration, i.e., the MVG configuration that yields $n^{\max }$ and $G_{n}^{\max }$ from Eqs (22) to (24), and for the MET configurations they are obtained from Eqs (25) and (29). In addition, the plotted values of $R$ are dictated by the MET system so they fall on the curve dictated by Eq. (25).

Examination of Figs 13, 14 and 15 leads to the main conclusion of this paper: the MVG configuration is more optimal than the MET configuration. Not only does the MVG configuration provide higher velocity gain, higher kinetic energy transfer efficiency, and lower system mass, it is effective over much larger ranges for $R$ - even for very small values of $R$. The improved performance of the MVG system is even more pronounced for lower values of $e$, which could be beneficial as the restitution of an actual shock amplifier might degrade over time. 


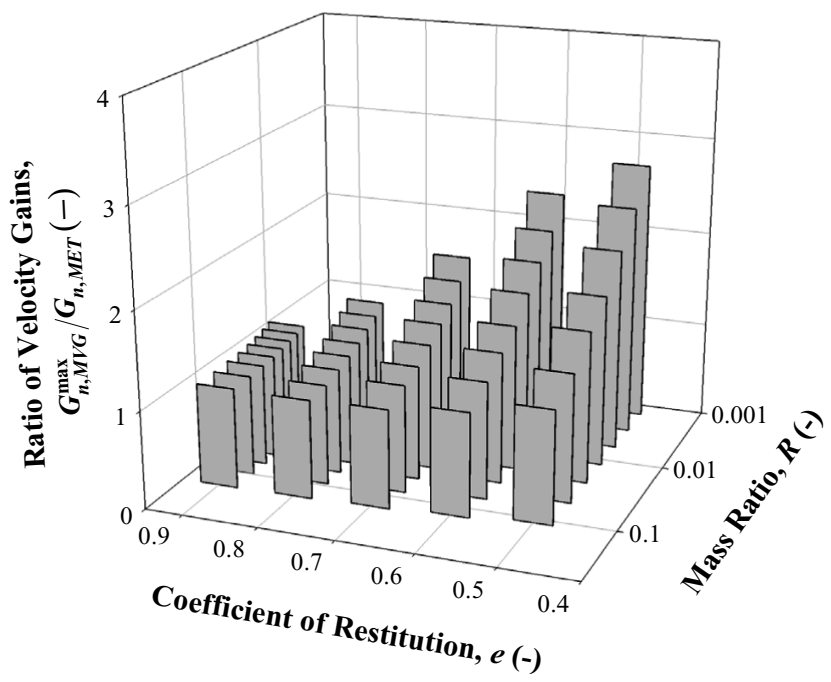

Fig. 13. 3D plot of ratio (MVG/MET) of velocity gains versus coefficient of restitution and mass ratio. The velocity gain for the MVG system is that of the optimal MVG system for the given $R, e$. (-) means non-dimensional.

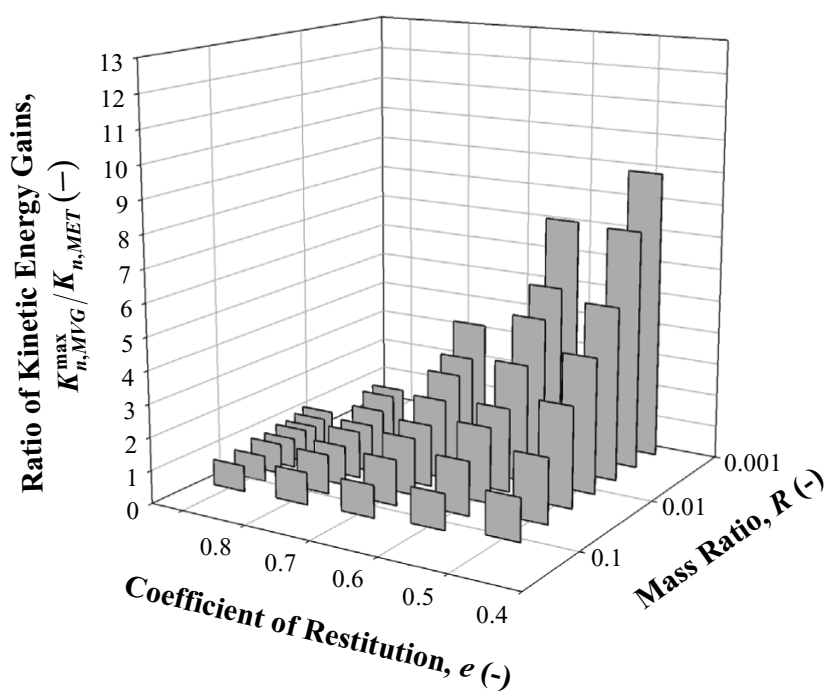

Fig. 14. 3D plot of ratio (MVG/MET) of kinetic energy transfer efficiencies for the top-most mass versus coefficient of restitution and mass ratio. The kinetic energy transfer efficiency for the MVG system is that of the optimal MVG system for the given $R, e$. (-) means non-dimensional.

To build up intuition supporting the above design guidelines, the performance of MVG and MET designs for a ten mass vertically-stacked shock amplifier with $R=0.012\left(m_{10}=0.1, m_{1}=8.4\right)$ and $e=0.8$ are plotted in more detail. Figure 16 plots the velocity gain for each of the masses in the MET system and for each of the masses in a three-mass, four-mass, and up to ten-mass MVG systems, to illustrate that the final velocity gain is higher for the MVG system in each case.

Figure 17 compares the mass distribution in the ten-mass MET system with the ten-mass MVG system and its optimal six-mass MVG counterpart to show how similar they turn out to be in the optimal MET and MVG systems. Although the total mass in both the MET and the six-mass MVG systems is about the same, the additional four masses of the former produce enough dissipation that its final velocity gain is significantly lower (about 23\%). The ten-mass MVG system has a slightly higher velocity gain than the MET system at the expense of having $50 \%$ additional total mass. 


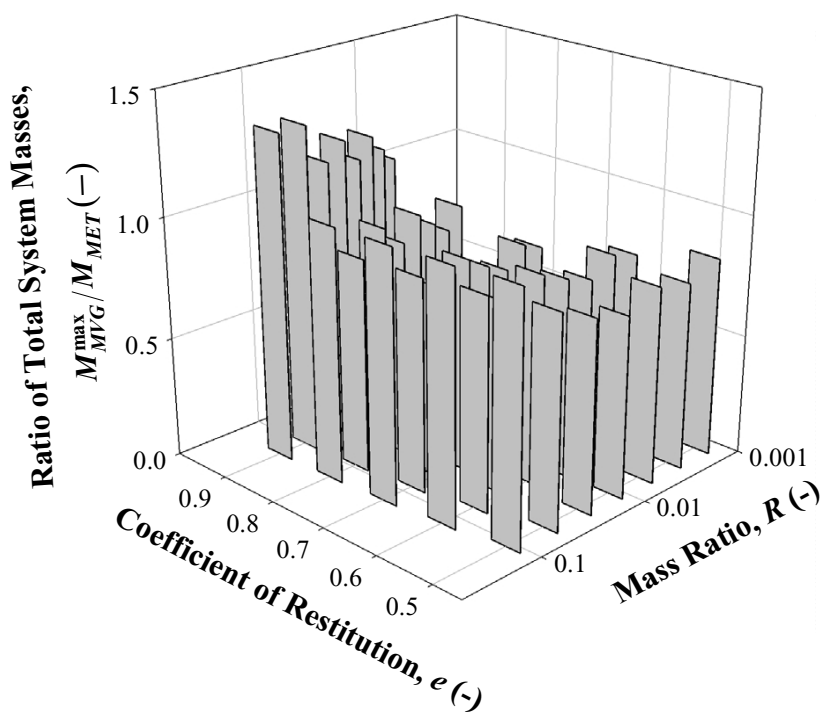

Fig. 15. 3D plot of ratio (MVG/MET) of total system masses versus coefficient of restitution and mass ratio. The total mass for the MVG system is that of the optimal MVG system for the given $R, e$. (-) means non-dimensional.

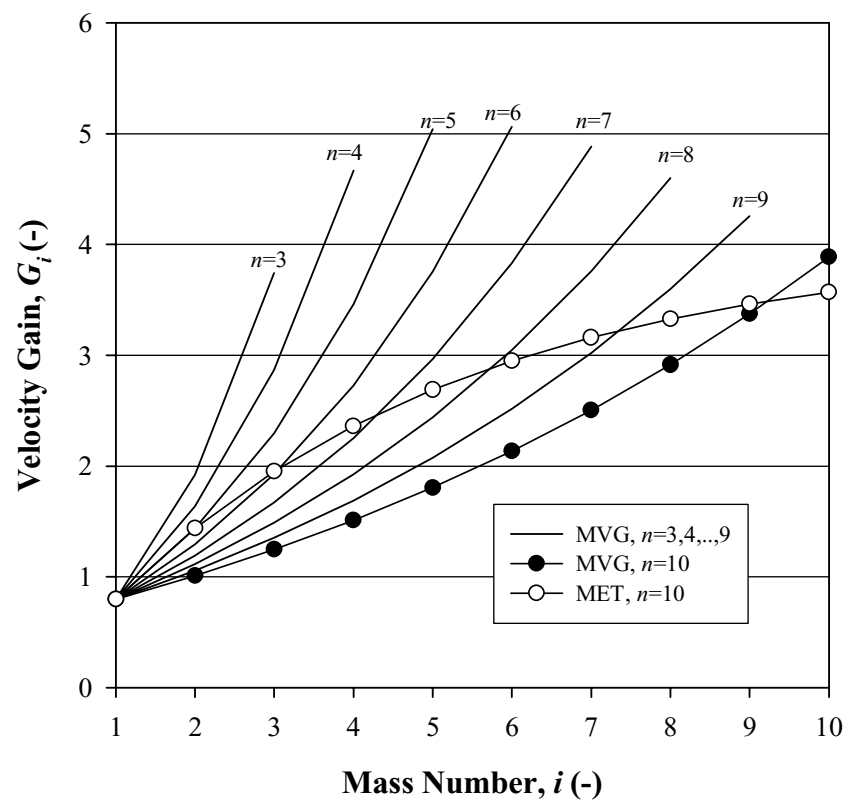

Fig. 16. Plot comparing vertically-stacked MVG systems (from three to ten masses) and the optimal ten-mass MET system for $e=0.8$ and $R=0.012$. In each system the velocity gain is plotted for every mass (i.e., velocity gain of every mass just after impact with a mass below it) to highlight its sequential growth. (-) means non-dimensional.

Finally, Fig. 18 gives a broader view of the design and performance metrics for MET-based and optimal MVGbased vertical shock amplification systems for $e=0.8$.

The guidelines presented in this section are general; the design of an actual shock amplifier should be determined after evaluating both MVG and MET models.

Actual shock amplifiers were built using both MET and MVG systems (see Fig. 19). The performance of the machine is being studied and its design optimized in subsequent versions. Construction and performance details of 


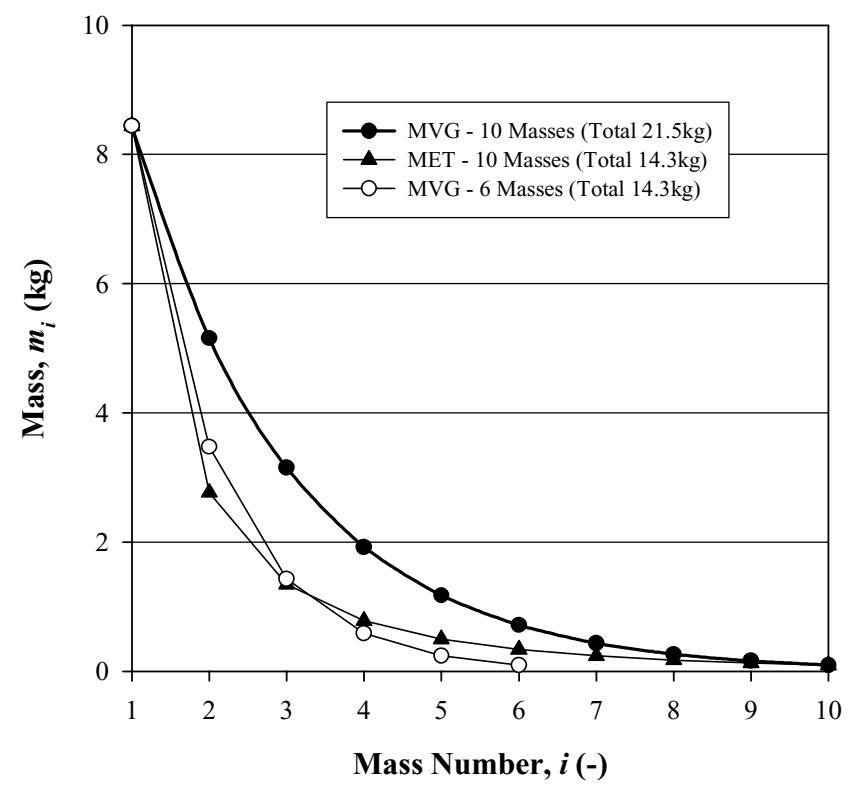

Fig. 17. Mass distributions for a system of 10 vertically-stacked masses comparing MVG and MET mass ratios (with $e=0.8$ ), and also showing the masses for the optimised MVG system of 6 masses. (-) means non-dimensional.

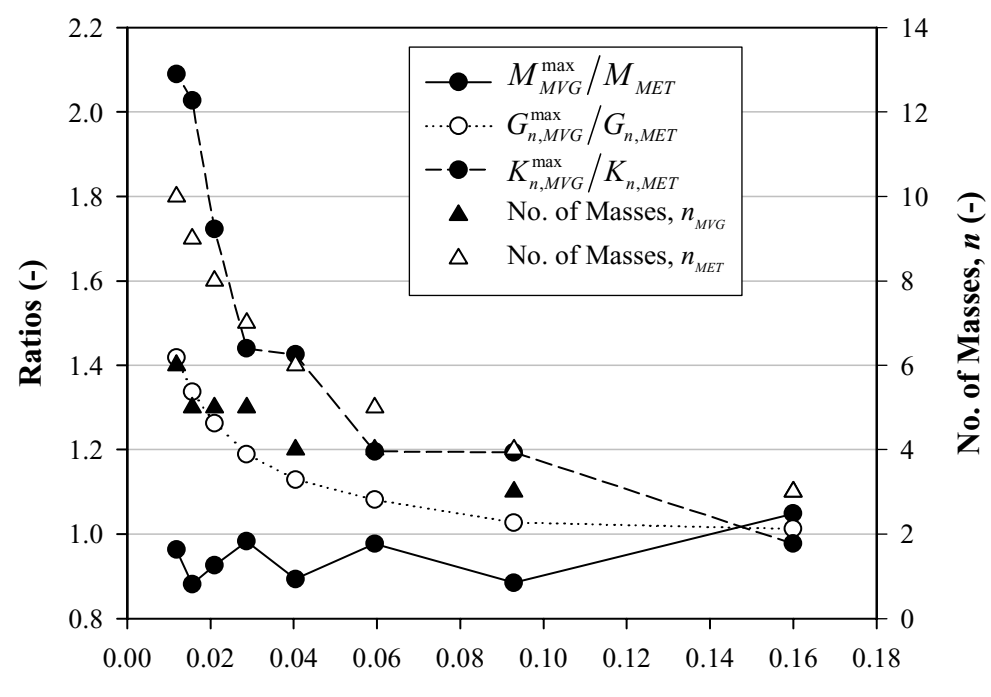

Mass Ratio, $R(-)$

Fig. 18. Comparison of total system mass, velocity gain and kinetic energy transfer efficiency for MET and MVG systems with $e=0.8$. Note that the number of masses for the MVG system has been optimised. (-) means non-dimensional.

these shock amplification machines, and comparisons with theory, will be published in upcoming papers.

\section{Discussion \& summary}

This paper examined the dynamics of velocity amplification through pair-wise collisions between multiple masses in a chain, with a view to developing useful machines. Although multiple collisions can be used both for velocity amplification and attenuation, the focus of the current work was on the design of shock amplification machines. All 


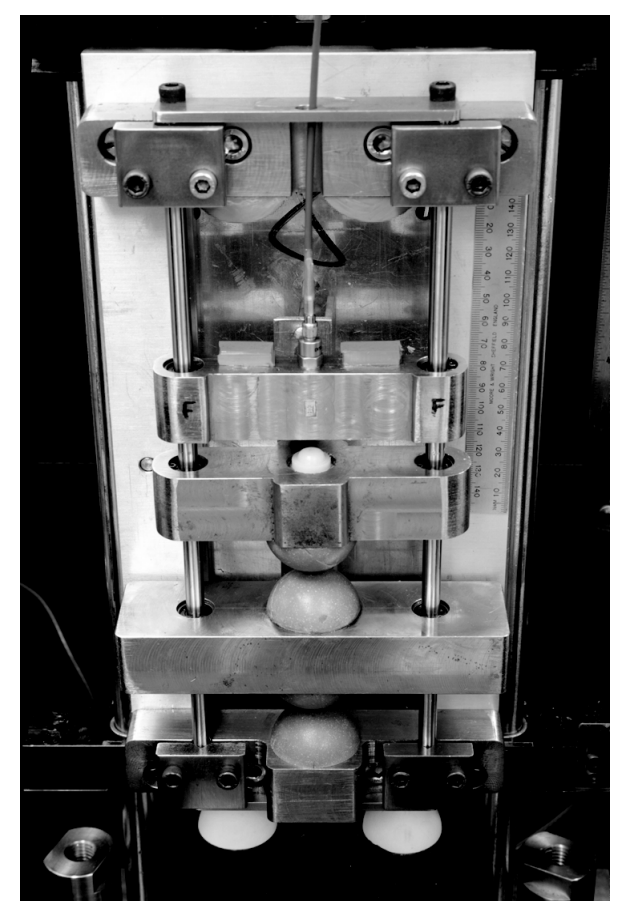

Fig. 19. Photograph of a four-mass vertically-stacked shock amplifier.

the analyses presented in the paper were based on simple but sufficient rigid body models - primarily those governing the momentum transfer and energy dissipation during impacts between two point masses or rigid spheres.

The dynamics of impact between two masses was examined in detail to show that a heavy mass impacting a light-enough-mass, with a sufficient coefficient of restitution, can cause a significant velocity amplification of the lighter mass. A special case of such impacts between two moving masses was identified, to be exploited in the design of shock amplifiers, where one of the masses becomes stationary after impact transferring all its momentum to the other mass. It was also proven why such maximum momentum and energy transferring impacts do not result in velocity amplification if one of the masses is stationary before impact.

Results from the two-body impact case were applied to investigate the performance of a shock amplifier that consists of a horizontal chain of monotonically decreasing masses, separated from each other, where all masses are stationary except the heaviest one. The pair-wise sequence of impacts that follow, starting with the heaviest mass impacting its neighbour, result in the lightest mass experiencing significant velocity amplification that is a function of the mass ratios in the chain and the coefficient of restitution. For a given mass ratio between the heaviest and the lightest mass and a specified number of intermediate masses in the chain, a constant mass ratio between the masses, such that each mass is the geometric mean between its two neighbours, maximizes the velocity gain of the lightest mass. Therefore the optimal mass ratio is given by a power law that is independent of the coefficient of restitution. The design of the shock amplifier can be optimized further by determining the unique number of masses, that does depend on the coefficient of restitution, to yield the highest velocity gain for the lightest mass.

The design and performance of the more natural - and common - shock testing configuration, consisting of a chain of monotonically decreasing masses (heaviest mass at the bottom) that is dropped from some height onto a high-restitution surface, was analysed in great detail. It was shown that all the general conclusions surrounding a design that uses the power-law based mass ratios described above are still valid. In particular it was shown that vertically-stacked shock amplifiers with an optimum number of masses with mass ratios governed by the power law, are ideal in the sense that they yield the highest velocity gain in systems with reasonably high coefficients of restitution. In addition, these shock amplifier designs are optimal for very large ranges of the mass ratio between the lightest mass (carrying the object under test) and the heaviest mass and can deliver velocity amplifications for coefficients of restitution that barely exceed 0.35 . 
A slightly different version of the vertically-stacked shock amplifier described above, based on the maximum energy transfer principle where all the masses come to rest after impact except the lightest one, was also analyzed. The mass ratios for such a shock amplifier depend on the coefficient of restitution. Although they require a coefficient of restitution of greater than 0.5 to produce any velocity amplification, for perfectly elastic collisions they produce a velocity gain that grows linearly with the number of masses in the chain.

The shock amplification configurations investigated in this paper are fairly simple, designs that would lead to straight-forward machines. If higher velocity amplification is desired than that attainable with the above machines, other features can be added. For instance in the vertically-stacked configuration, the restitutive surface that the masses drop onto could be 'active', i.e., it adds net energy to the system; the initial velocity of the uppermost mass could be made higher than that of the other masses, etc. The analysis of the above scenarios is very much in the scope of the methods presented in this paper.

The dynamics principles expounded in this paper, and the design guidelines, can be used for multiple purposes: building launchers and shock amplifiers, significantly decreasing the size or the form factor of current shock-testing machines, for building protective armour and energy absorbers, for increasing the efficiency of energy harvesters, etc. Particularly in the area of shock testing of emerging opto-electronic components like MEMS \& nano-scale devices, that are inherently very rugged or being used in space and military applications, the shock amplifiers described in this paper provide a low-cost, versatile, safe, detailed, precise and observable alternative to the currently practised ballistic and other methods.

In upcoming papers, the design, construction and performance details of shock amplification machines built using the above principles will be presented. Challenges around the shaping of very-high acceleration shock pulses will also be addressed.

\section{Acknowledgments}

The authors wish to acknowledge support from the Centre for Telecommunications Value-Chain Research (CTVR), which received funding from Science Foundation Ireland under grant number 03/CE3/I405. The involvement of Bell Labs Ireland researchers was partially supported by a grant from the Industrial Development Agency (IDA) Ireland. Finally, acknowledgement is also given to IRCSET (Irish Research Council for Science, Engineering and Technology) for their support of this work.

\section{References}

[1] V. Bateman, F. Brown and N. Davie, Use of a Beryllium Hopkinson Bar to Characterize a Piezoresistive Accelerometer in Shock Environments, Journal of the Institute of Environmental Sciences 39(6) (1996).

[2] R.M. Brach, Mechanical Impact Dynamics - Rigid Body Collisions, John Wiley \& Sons, 1991.

[3] W. Goldsmith, The Theory and Physical Behaviour of Colliding Solids, Edward Arnold Publishers Ltd., London, 1960.

[4] S. Goyal, J. Papadopoulos and P. Sullivan, Shock Protection of Portable Electronic Products: Shock Response Spectrum, Damage Boundary Approach, and Beyond, Shock and Vibration 4(3) (1997), 169-191.

[5] S. Goyal, J. Papadopoulos and P. Sullivan, The Dynamics of Clattering I: Equation of Motion and Examples, Journal of Dynamic Systems Measurement Control 120 (1998), 83-93.

[6] J.B. Hart and R.B. Herrmann, Energy Transfer in One-Dimensional Collisions of Many Objects, American Journal of Physics 36 (1968), $46-48$.

[7] W.G. Harter, Velocity Amplification in Collision Experiments Involving Superballs, American Journal of Physics 39 (1971), $656-663$.

[8] J.D. Kerwin, Velocity, Momentum, and Energy Transmissions in Chain Collisions, American Journal of Physics 40 (1972), $1152-1157$.

[9] P. Patrício, The Hertz Contact in Chain Elastic Collisions, American Journal of Physics 72(12) (2004), 1488-1491.

[10] T. Pöschel and N. Brilliantov, Extremal Collision Sequence of Particles on a Line: Optimal Transmission of Kinetic Energy, Physical Review E 63 (2001), 021505.

[11] S. Zhang-Shu, Survey on High-G Testing Methodology, 2005, [online], available: http://www.empf.org/empfasis/june05/g0605.htm [accessed 10 August 2006]. 

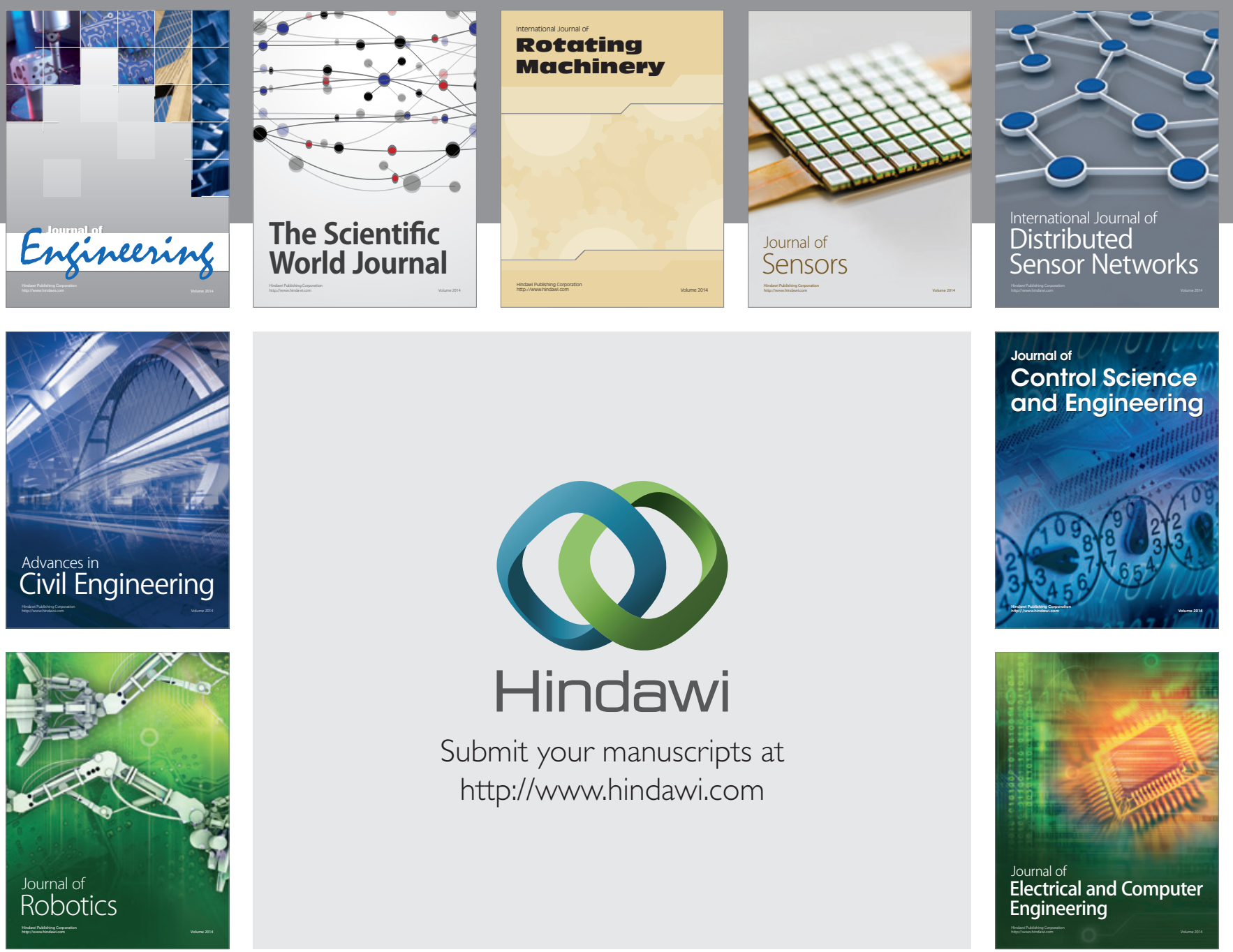

Submit your manuscripts at

http://www.hindawi.com
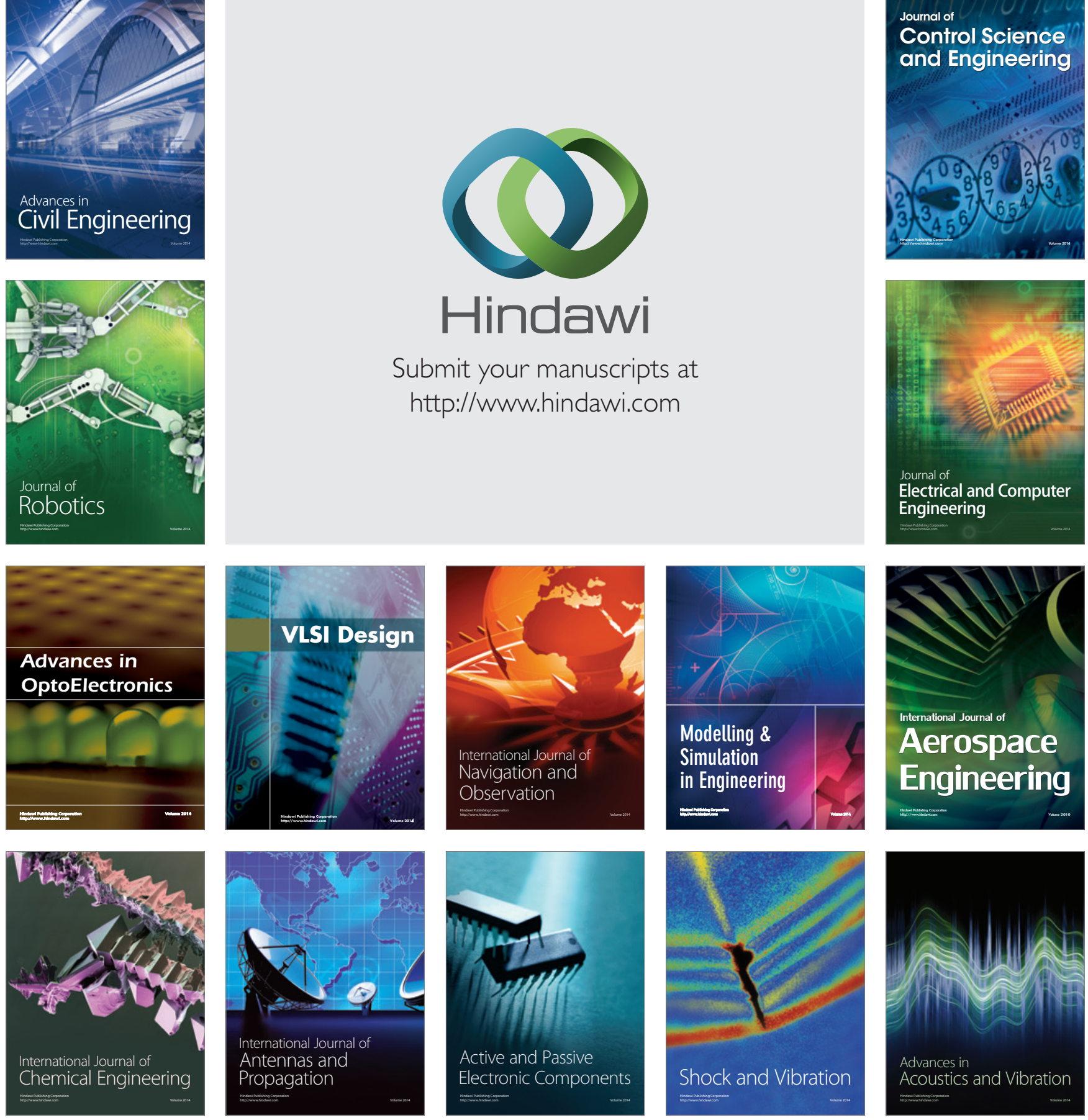